\title{
Model prediction of subendocardial perfusion of the coronary circulation in the presence of an epicardial coronary artery stenosis
}

\author{
Jeroen P. H. M. van den Wijngaard · Christina Kolyva • \\ Maria Siebes · Jenny Dankelman · Martin J. C. van Gemert • \\ Jan J. Piek · Jos A. E. Spaan
}

Received: 22 October 2007 / Accepted: 23 January 2008/Published online: 14 February 2008

(C) International Federation for Medical and Biological Engineering 2008

\begin{abstract}
The subendocardium is most vulnerable to ischemia, which is ameliorated by relaxation during diastole and increased coronary pressure. Recent clinical techniques permit the measuring of subendocardial perfusion and it is therefore important to gain insight into how measurements depend on perfusion conditions of the heart. Using data from microsphere experiments a layered model of the myocardial wall was developed. Myocardial perfusion distribution during hyperemia was predicted for different degrees of coronary stenosis and at different levels of Diastolic Time Fraction (DTF). At the reference DTF, perfusion was rather evenly distributed over the layers and the effect of the stenosis was homogenous. However, at shorter or longer DTF, the subendocardium was the first or last to suffer from shortage of perfusion. It is therefore concluded that the possible occurrence of
\end{abstract}

J. P. H. M. van den Wijngaard $(\bowtie) \cdot$ C. Kolyva · M. Siebes ·

J. A. E. Spaan

Department of Medical Physics, Academic Medical Center,

University of Amsterdam, Amsterdam, The Netherlands

e-mail: J.P.vandenwijngaard@amc.uva.nl

C. Kolyva

Institute for Bioengineering, Brunel University London,

London, England

J. Dankelman

Department of BioMechanical Engineering,

Delft University of Technology, Delft, The Netherlands

M. J. C. van Gemert

Laser Center, Academic Medical Center,

University of Amsterdam, Amsterdam, The Netherlands

J. J. Piek

Department of Cardiology, Academic Medical Center,

University of Amsterdam, Amsterdam, The Netherlands subendocardial ischemia at exercise is underestimated when heart rate is increased and DTF is lower.

Keywords Coronary reserve $\cdot$ Steal $\cdot$ Heart model · Regional conductance $\cdot$ Diastolic time fraction

\section{Introduction}

Myocardial tissue depends for its perfusion on a stenotic coronary artery and may become deprived of blood supply with increasing stenosis resistance (SR), leading to ischemia and ultimately to cell death. In the presence of a mild stenosis, the normal auto regulatory control of the distal vascular bed compensates for the pressure loss over the stenosis. However, with a severe stenosis, this autoregulatory vasodilatation may be exhausted at moderate exercise or even at rest.

Several diagnostic tests have been developed to support clinical decision making with respect to treatment of an epicardial stenosis by percutaneous transluminal coronary angioplasty, PTCA, or stent placement. These tests are based on the measurement of distal pressure, flow velocity or combination of both by applying a so-called guide wire (tip $0.3 \mathrm{~mm}$ diameter) having a pressure and/or velocity sensor at its tip [16]. These measurements result in indices like coronary flow velocity reserve (CFVR), fractional flow reserve (FFR) or SR $[15,18]$. These indices provide a threshold level value below or above which the cardiologist will treat the stenosis. However, the fact that the perfusion of the myocardium differs over the various myocardial layers depending on the proximity of the left ventricular cavity is neglected in the decision protocols.

Presently, many techniques are being developed to measure perfusion differences over the myocardial wall. 
MRI, especially, is a promising technique in this respect. These measurements confirm the earlier findings in animals that the subendocardium is particularly vulnerable to ischemia $[1,5]$. The reason for this is that myocardial contraction has an impeding effect on blood flow, especially in the inner layer of myocardial tissue while in the subepicardium or outer layer this compression effect is minimal [22]. These new image-based modalities to measure the perfusion distribution provide great new opportunities to evaluate the physiological impact of an epicardial stenosis on tissue perfusion. The simplest stress test to apply is the local or systemic injection of adenosine, which mimics the perfusion condition when autoregulatory flow adaptation is exhausted. However, the microvascular resistance in the subendocardium at hyperemia strongly depends on the pressure distal to the stenosis and the diastolic time fraction (DTF) [8], which strongly depends on HR [3]. For a proper interpretation of an adenosine test it is therefore necessary to have insight into how the distal pressure of a stenosis and DTF affect the perfusion distribution in the wall of the beating heart.

In the present model study we apply data of animal studies [8] to predict changes in myocardial perfusion distribution during an adenosine stress test. This bears clinical importance since changes in DTF and perfusion pressure are not taken into account when applying this adenosine test in the clinical setting. We hypothesized that above a certain HR adenosine would underestimate the subendocardial perfusion as a result of steal induced by subepicardial vasodilation. Furthermore, we expect that subendocardial perfusion will be underestimated for low HR. We further define the conditions of coronary pressure and DTF, where perfusion is expected to be homogenously distributed over the different layers, and relate this condition to threshold values of currently used clinical indices to quantify the significance of a coronary stenosis.

\section{Methods}

\subsection{Arrangement of myocardium in parallel layers}

In the model, the heart is assumed to be a hollow muscle divided into three parallel myocardial layers as previously used for data representation [8], i.e., the subepicardial, midmyocardial and the subendocardial layer as depicted in Fig. 1a. Each of the myocardial tissue layers possesses an autoregulatory resistance so that flow in each layer is controlled within the normal range. The reference condition of the model was defined by a perfusion pressure of $90 \mathrm{mmHg}$ and a venous pressure of $5 \mathrm{mmHg}$. We assumed a human reference heart weighing $300 \mathrm{~g}$, and the coronary artery supplies $100 \mathrm{~g}$ of tissue, subdivided into three layers of equal weight. In the reference condition there is no coronary stenosis and DTF is taken as 0.5. Collaterals are not included in the model.

Each tissue element is represented by a total resistance, $R_{\mathrm{tot}}$, which is the sum of a series of two resistances, one representing hyperemic resistance, $R_{\text {hyp }}$ and the other autoregulatory resistance, $R_{\text {auto }}$ (see Fig. $1 \mathrm{~b}$ ).

\subsection{Parameterization of the model}

Layer blood flow conductance is expressed as $\mathrm{ml} \mathrm{min}^{-1} \mathrm{~g}^{-1}$ $\mathrm{mmHg}^{-1}$ and for the hyperemic state, it depends on (DTF) and perfusion pressure (Pc) of the myocardial layer [8]. This data set results in the hyperemic conductances for the model. Bache determined flow reserve for the different layers at different heart rates but only one value for Pc [1]. We used these regional flow reserves $(4.36,4.28$ and 4.02 for the subepicardial, midmyocardial and subendocardial layer, respectively), at HR $=100$ for estimating the autoregulatory resistances. In our model however, the flow reserve in hyperemia is slightly lower as a consequence of small pressure decrease across the coronary artery. In case the dependency of the hyperemic resistances on DTF and $\mathrm{Pc}$ is equal for all layers, the autoregulatory resistances of all layers would be equal as well. However, as shown by Fokkema, the hyperemic resistances have a layer-specific response to a change in pressure. Therefore, the autoregulatory resistances and the pressures proximal to the hyperemic resistance become layer-specific as well.

Since the mean goat myocardial conductance as found by Fokkema, is somewhat increased as compared to human myocardial conductance [20], e.g., approximately 0.014 versus $0.0083 \mathrm{ml} \mathrm{min}^{-1} \mathrm{~g}^{-1} \mathrm{mmHg}^{-1}$ in humans assuming a $225 \mathrm{ml} \mathrm{m^{-1 }}$ flow, $90 \mathrm{mmHg}$ and a 300 -g myocardium, the myocardial conductance of each layer as given by Fokkema was reduced by 40\%. In Table 1 an overview of the conductance of each myocardial layer is given.

Subsequently, from the normal flow and the fractional flow increase it is possible to obtain the distribution of resistance between the autoregulatory and the hyperemic resistances. The hyperemic resistance depends on DTF, coronary pressure and the myocardial layer. The autoregulatory resistances are calculated from pressure and flow that match the assumed local oxygen demand and the layer-specific hyperemic resistances. These pressure and flow data result in $R_{\text {tot }}$ as long as $R_{\text {tot }}>R_{\text {hyp }}, R_{\text {auto }}=$ $R_{\mathrm{tot}}-R_{\mathrm{hyp}}$. In case $R_{\mathrm{tot}} \leq R_{\mathrm{hyp}}$, the corresponding autoregulatory resistance is 0 and $R_{\mathrm{tot}}$, is made equal to $R_{\mathrm{hyp} .}$. In that case, the flow will be lower then needed according to demand and flow will follow from the resistance value and 


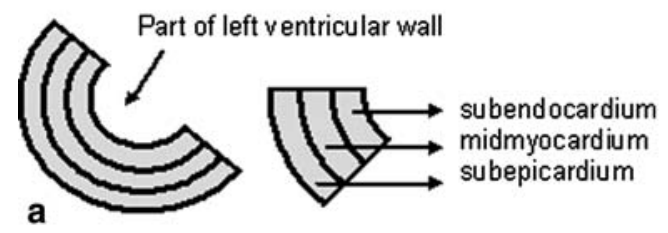

Fig. 1 a Schematic illustration of the arrangement of the left ventricle wall into a series of concentric tissue slices organized as parallel layers. b Arrangement of myocardial layers, stenosis resistance and autoregulation resistances. Here Paorta is the aortic pressure, $P c$ is coronary pressure distal of an epicardial stenosis and

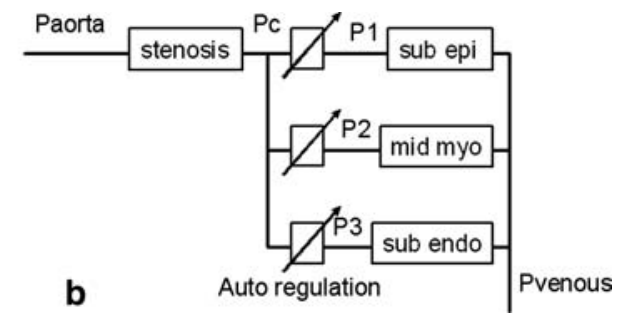

Pvenous denotes the venous pressure. The autoregulatory resistances of each tissue layer are denoted by variable resistances, whereas the hyperemic resistances are denoted by their respective name. $P 1, P 2$ and $P 3$ are the pressures that perfuse the hyperemic resistance

Table 1 Parameters used in the model

\begin{tabular}{llcc}
\hline Myocardial layer & $\begin{array}{l}\text { Hyperemic conductance } \\
(\mathrm{ml} / \mathrm{min} / \mathrm{g} / \mathrm{mmHg})\end{array}$ & $\begin{array}{l}\text { Assumed maximal flow } \\
\text { in hyperemia }(\mathrm{ml} / \mathrm{min})\end{array}$ & $\begin{array}{l}\text { Normal flow per myocardial } \\
\text { layer }\end{array}$ \\
\hline Subepicardium & $0.0149-0.0137 \mathrm{DTF}+0.0003 \mathrm{Pc}^{\mathrm{a}}$ & 98.5 & 22.8 \\
Midmyocardium & $-0.0288+0.0756 \mathrm{DTF}+0.00029 \mathrm{Pc}$ & 99.6 & 23.5 \\
Subendocardium & $-0.0618+0.118 \mathrm{DTF}+0.00044 \mathrm{Pc}$ & 104.5 & 26.3 \\
Total coronary flow & & 302.6 & 72.6
\end{tabular}

a Subepicardial conductance was obtained from data by Fokkema et al. represented in their Fig. 2b. Please note that subepicardial conductance is only weakly influenced by subepicardial pressure and DTF

b The reference DTF is assumed as 0.5 , normal aortic and venous pressures are assumed 90 and $5 \mathrm{mmHg}$ respectively

pressure. Administration of adenosine in the normal situation sets the resistance of the autoregulation to 0, i.e., causes maximal flow in each layer.

\subsection{Modeling of coronary stenosis}

Modeling the influence of an epicoronary stenosis was done as previously $[14,15]$. In brief, the combined SR results from viscous friction according to Poiseuille's law and the resistance resulting from convective acceleration within a narrowed flow channel and exit pressure loss upon diameter increase according to Bernoulli's law [14, 21]. The total SR as a consequence of these combined effects is

$R_{\text {Stenosis }}=R_{\text {Viscous }}+R_{\text {Bernoulli }}$

where [14]:

$R_{\mathrm{Viscous}}=C_{1} k_{\mathrm{v}} \frac{\mu}{D_{0}^{3}}$

$R_{\text {Bernoulli }}=C_{2} k_{\mathrm{t}} \frac{\rho}{2 D_{0}^{4}}\left(\frac{A_{0}}{A_{1}}-1\right)^{2} \cdot Q_{\text {Stenosis }}$

where $C_{1}$ and $C_{2}$ represent dimensionless constants, $\mu$ is the blood viscosity, $\rho$ is the blood density, $D_{0}$ the normal coronary artery diameter, $A_{0}$ is the normal coronary artery area and $A_{1}$ is the minimal area inside the stenosed segment. $Q_{\text {Stenosis }}$ represents the coronary flow through the stenosis. Both coefficients $k_{\mathrm{v}}$ and $k_{\mathrm{t}}$ depend on the stenosis area and the inlet and exit angles of the convergent and divergent parts of the stenosis [14]. In case there is no coronary stenosis, $A_{0}=A_{1}$ and $R_{\text {Bernoulli }}$ becomes 0 , and the pressure loss over the coronary artery is determined by $R_{\text {Viscous }}$ only.

\begin{tabular}{ll}
\hline Parameter & Description and units \\
\hline$C_{1}$ & Constant (dimensionless) $4 /(\pi \times 60 \times 1333.33)$ \\
$C_{2}$ & Constant (dimensionless) $16 /\left(\pi^{2} \times 60^{2} \times 1333.33 \times 2\right)$ \\
$A_{0}, A_{1}$ & Area of vessel without or with stenosis $\left(\mathrm{cm}^{2}\right)$ \\
$D_{0}, D_{\text {min }}$ & Normal and minimal vessel diameter $(\mathrm{cm})$ \\
$L$ & Stenosis length $(\mathrm{cm})$ \\
$k_{\mathrm{v}}$ & Viscous coefficient (dimensionless) \\
$k_{\mathrm{t}}$ & Turbulence coefficient (dimensionless) $1.21+0.08 \cdot L / D_{0}$ \\
$\mu$ & Blood viscosity $0.036($ Poise $)$ \\
$\rho$ & Blood density $1.06\left(\mathrm{~g} \mathrm{ml}{ }^{-1}\right)$ \\
$Q_{\text {Stenosis }}$ & Flow through the stenosis $\left(\mathrm{ml} \mathrm{min}{ }^{-1}\right)$ \\
\hline
\end{tabular}




\begin{tabular}{lll}
\hline \% Diameter stenosis & $D_{\min }(\mathrm{cm})$ & $k_{\mathrm{v}}$ \\
\hline 0 & 0.30 & 19.9 \\
20 & 0.24 & 48.6 \\
30 & 0.21 & 82.9 \\
40 & 0.18 & 154 \\
50 & 0.15 & 318 \\
60 & 0.12 & 778 \\
70 & 0.09 & 2,457 \\
80 & 0.06 & 12,440 \\
90 & 0.03 & 199,040 \\
\hline
\end{tabular}

\subsection{Calculation of myocardial resistances, pressures} and flows in case of coronary stenosis

In case of a coronary stenosis, the pressure loss resulting from the flow through the stenosis reduces the coronary pressure for perfusion of the segments. To maintain normal perfusion, the autoregulatory resistance $R_{\text {auto }}$ per segment is reduced except when it becomes 0 . In that case, autoregulation resistance is exhausted, and myocardial flow cannot be enhanced by a further reduced autoregulatory resistance.

In the model, all resistances are calculated first on the basis of perfusion pressures in the myocardial layers. Subsequently, the total resistance of the SR connected with the three parallel myocardial layers is determined. Then, from the aortic and venous pressure gradient, total tissue flow can be obtained. From the resistances of the myocardial layers, the flow per layer and thus the tissue perfusion can be obtained. In case of a lower or higher tissue perfusion than normal, the autoregulation resistance of the myocardial layer is reduced or increased and the procedure is repeated. The iteration is terminated when the difference between the flow for each layer with the normal flow is less than $10^{-3}$ or the autoregulation of the specific layer is exhausted. In case of simulated adenosine administration, the autoregulation resistances are set to 0 .

\subsection{Definition of fractional flow reserve and coronary flow velocity reserve}

In this study, the influence of collateral flow is ignored and FFR is defined as

$\mathrm{FFR}=\frac{Q_{\mathrm{S}}}{Q_{\mathrm{N}}}=\frac{\left(P_{\mathrm{d}}-P_{\mathrm{b}}\right)}{\left(P_{\mathrm{a}}-P_{\mathrm{b}}\right)}$

with $Q_{\mathrm{S}}$ the maximum flow in hyperemia with stenosis and $Q_{\mathrm{N}}$ the maximum flow in hyperemia in the absence of a stenosis. Here, $P_{\mathrm{b}}$ is the coronary outflow pressure, which can be obtained from the intercept of the flow pressure curve with the abscissa. In experiments, $P_{\mathrm{b}}$ actually is often higher than $P_{\mathrm{v}}$ due to partial collapse of the coronary microcirculation. In our study, however, we assumed $P_{\mathrm{b}}=0$.

The CFVR is defined as the ratio of the maximal $V_{\text {Max }}$ and normal $V_{\mathrm{N}}$ blood flow velocities. This can be measured clinically using a Doppler flow velocity wire. The coronary flow reserve (CFR) was developed to describe the fractional increase in coronary blood flow to the heart in case of increased oxygen demand from the heart. In our study however, we will use the ratio of maximal hyperemic and normal blood flow, so that the CFVR is approximated by the CFR as

$\mathrm{CFVR}=\frac{V_{\mathrm{M} a x}}{V_{\mathrm{N}}} \approx \frac{Q_{\mathrm{Max}}}{Q_{\mathrm{N}}}$

\subsection{Simulations}

The pressure loss across a coronary stenosis is simulated for different stenosis severities. Simulations were done for the normal controlled situation with intact autoregulation and varying DTF and coronary pressure through stenosis severity. In addition, these simulations were repeated for the hyperemic situation with absent autoregulation. The level of autoregulation as dependent on DTF is obtained, as well as the DTF yielding equal endocardial and epicardial flow, defined as $\mathrm{DTF}_{1}$, is simulated as a function of coronary pressure. Finally, the increase of flow demand by exercise and the influence of DTF on FFR are illustrated.

\section{Results}

\subsection{Pressure loss across a coronary stenosis}

The influence of changes in DTF on the subendocardial, midmyocardial and subepicardial flows can be investigated for different stenoses severities. For these simulations we chose stenoses so that for reference values $\mathrm{DTF}=0.5$ and PAorta $=90 \mathrm{mmHg}$ FFR resulted in the following values: FFR $=1$, FFR $=0.75$ and FFR $=0.5$ These FFR values correspond to 0,56 and $72 \%$ diameter occlusion of the vascular lumen, respectively. The pressure loss for these three stenoses as a function of coronary flow is given in Fig. 2.

\subsection{Myocardial layer flow with coronary stenosis}

Flow predictions for the different layers for increasing stenosis degree in the presence of autoregulatory control are illustrated in Fig. 3 for three DTF values. On the $x$-axis 


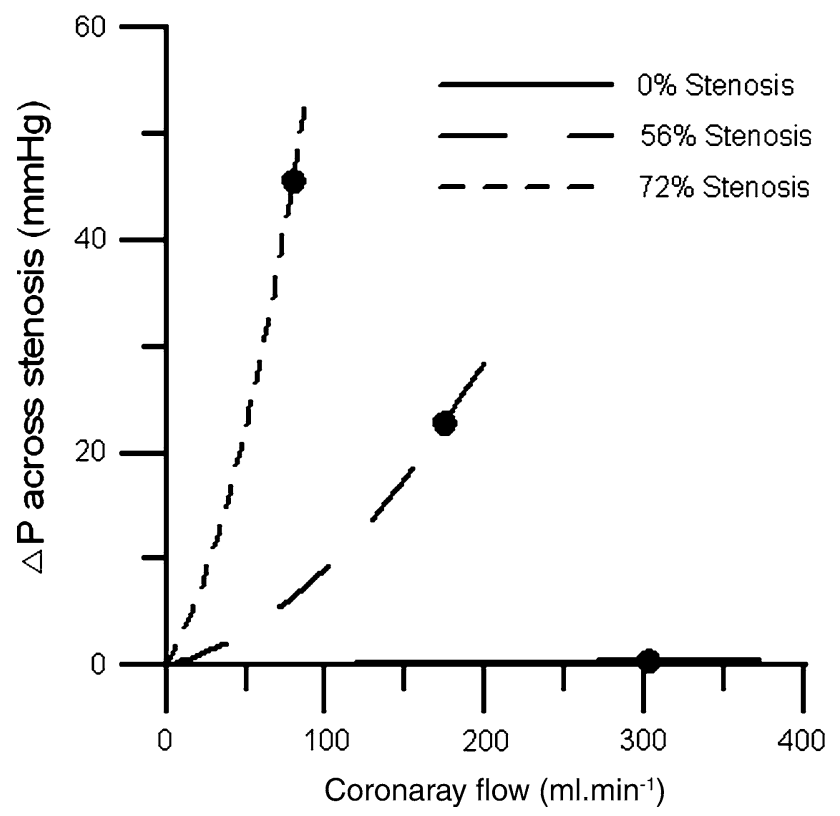

Fig. 2 Pressure loss across the stenosis as a function of coronary flow. The black dots indicate the pressure drop where FFR equals 1, 0.75 and 0.5 , respectively, for the reference conditions of DTF and aortic pressure

the distal coronary pressure $(\mathrm{Pc})$ is plotted, which is reduced by the increasing stenosis degree. Depending on the layer and DTF, flow is maintained at the prescribed value up to a certain threshold of pressure below which the autoregulatory response is exhausted and flow drops. The subendocardial hyperemic resistance is strongly affected by DTF while subepicardial hyperemic resistance is rather independent of DTF and pressure. Please note that for the reference condition $(\mathrm{DTF}=0.5$, no stenosis and Paorta $=$ $90 \mathrm{~mm} \mathrm{Hg}$ ), myocardial flow at the subendocardium is higher than subepicardium for low stenosis values.

The dependence of subendocardial perfusion on DTF is such that for DTF values smaller than the reference value autoregulatory response is exhausted earlier than in the subepicardium, but for larger DTF values the opposite effect is found. In any case subendocardial underperfusion will occur earlier at low coronary pressure and at a higher HR (smaller DTF).

Figure $4 \mathrm{a}$ and $\mathrm{b}$ demonstrates the hyperemic pressure flow relation during pharmacological vasodilation for the three layers. Panel A for the reference DTF and all three layers, Panel B for the three DTF's studied but only subepicardium and subendocardium. The curves depicted in this figure correspond to the trajectories of relationships in Fig. 3 where autoregulation was exhausted. The pressureflow relations are convex to the pressure axis, indicating that with decreasing pressure, myocardial hyperemic resistance is increased. As can be seen in Fig. $4 b$, the model predicts a shift of the flow pressure relations to the right with decreasing values of DTF but to the left with decreasing DTF. For reference the average mean flow for the case of autoregulation is shown as well. Also this figure demonstrates that that subendocardial reserve is exhausted earlier at higher heart rate and for lower SR.

\subsection{Myocardial autoregulation resistance}

Myocardial autoregulation resistances in the model are adjusted such that myocardial layer flow tends to remain normal, independent of changes in a range of coronary pressures. Autoregulation resistances decrease with decreasing perfusion pressure as with epicoronary stenosis. Since the hyperemic resistances depend on DTF, a reduced DTF may lead to a higher hyperemic resistance and consequently a lower level of autoregulation resistance to maintain normal prescribed flow. Autoregulatory resistances as a function of DTF in the absence of coronary stenosis at reference arterial pressure are depicted in Fig. 5. It is shown that subepicardial autoregulatory resistance (black column) is relatively uninfluenced by DTF, but for midmyocardial and subendocardial layers (grey columns), these resistances are lower at lower DTF
Fig. 3 a Left and b right. a Model outcomes for coronary pressure and myocardial flow per layer for the different myocardial layers. b Model outcomes for subepicardial and subendocardial flow for different values of DTF $(0.4,0.5$ and 0.6)
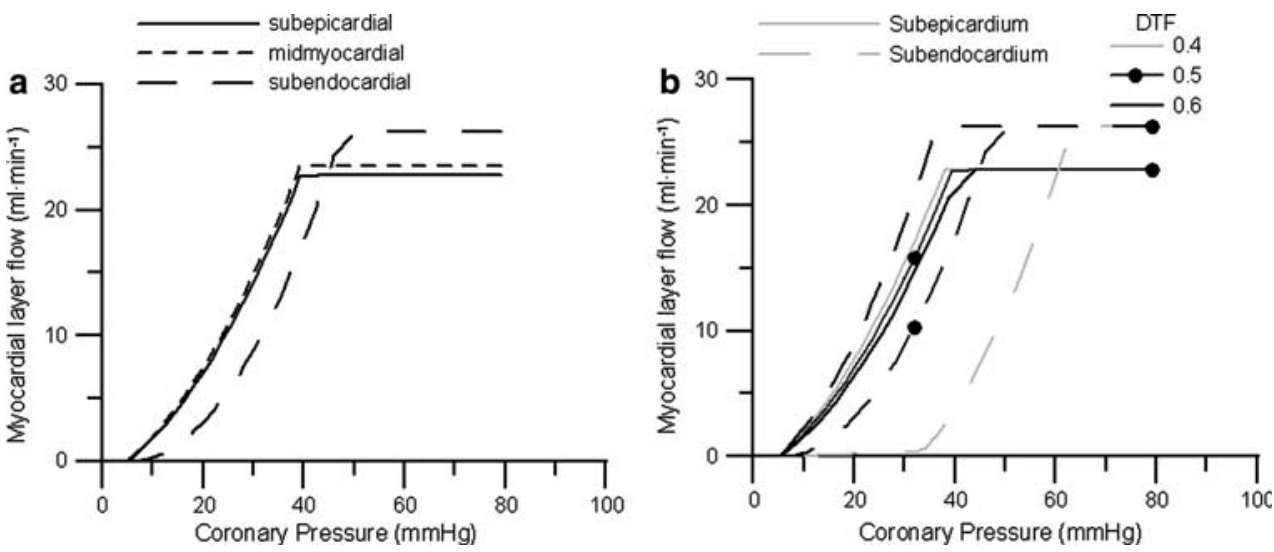
Fig. 4 a Left and b right. a Myocardial layer flow expressed as the percentage of normal flow as a function of coronary pressure for $\mathrm{DTF}=0.5$. b Subepicardial and subendocardial flow per layer as a function of coronary pressure. As can be seen from the level of flow increase, subendocardial layer conductance strongly depends on DTF $(0.4,0.5$ and 0.6$)$
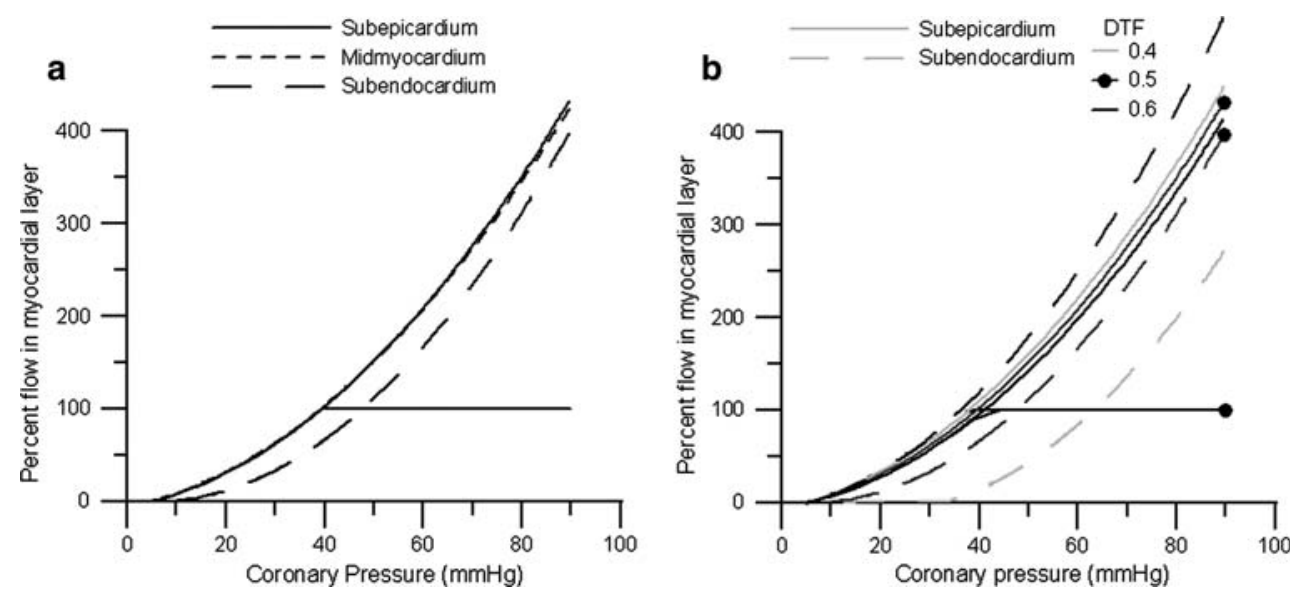

because more dilatory compensation is needed for the increased hyperemic resistance. This implies that these myocardial layers have a smaller range of autoregulatory compensation to correct for a pressure drop over a stenosis. Note that the subendocardium and midmyocardium are better protected from low coronary pressure for larger values of DTF.

\subsection{Comparison of endocardial and epicardial flow}

With intact autoregulation and without stenosis the endocardial and epicardial flow ratio equals 1.15 and is the result of autoregulatory compensation in each layer. In the hyperemic state however, this endo/epi ratio will in general be different from 1.15 but a $\mathrm{DTF}_{1}$, can be defined as function of coronary pressure for which the endo/epi range is 1 . This relationship between $\mathrm{DTF}_{1}$ and coronary pressure is depicted in Fig. 6. Since decrease in coronary perfusion pressure corresponds to higher increase in the hyperemic

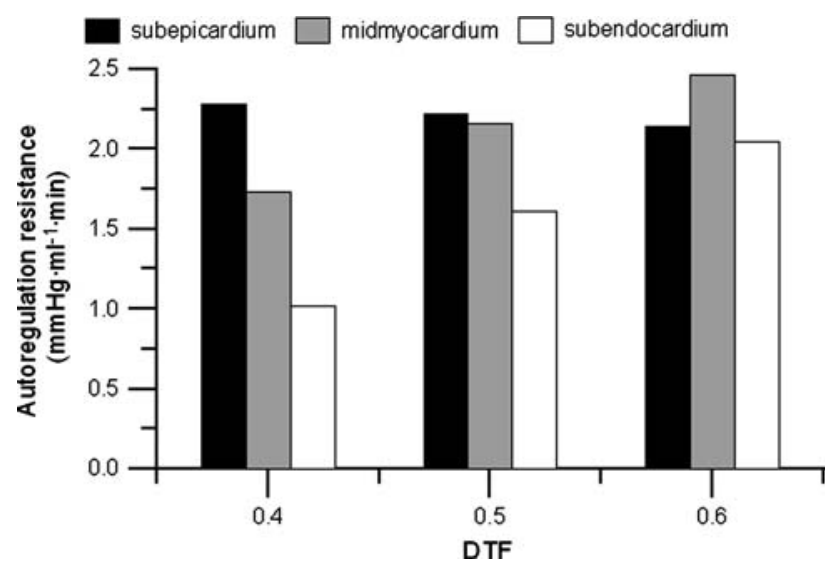

Fig. 5 Autoregulation resistances in the absence of a coronary stenosis of the myocardial layers as a function of DTF subendocardial resistance, a higher DTF (lower HR) is required for preservation of even flow distribution. The area below this curve indicates the DTF for which hyperemic perfusion in subendocardium is lower than in the subepicardium. For higher DTF values it has the opposite effect.

\subsection{Flow distribution depending DTF and coronary} stenosis

The effect of SR is demonstrated in Fig. 7a-c, where for each stenosis flow distribution at autoregulatory and hyperemic conditions are shown for three different DTF values. In the absence of a coronary stenosis and for the $56 \%$ stenosis autoregulatory flow is not affected by DTF,

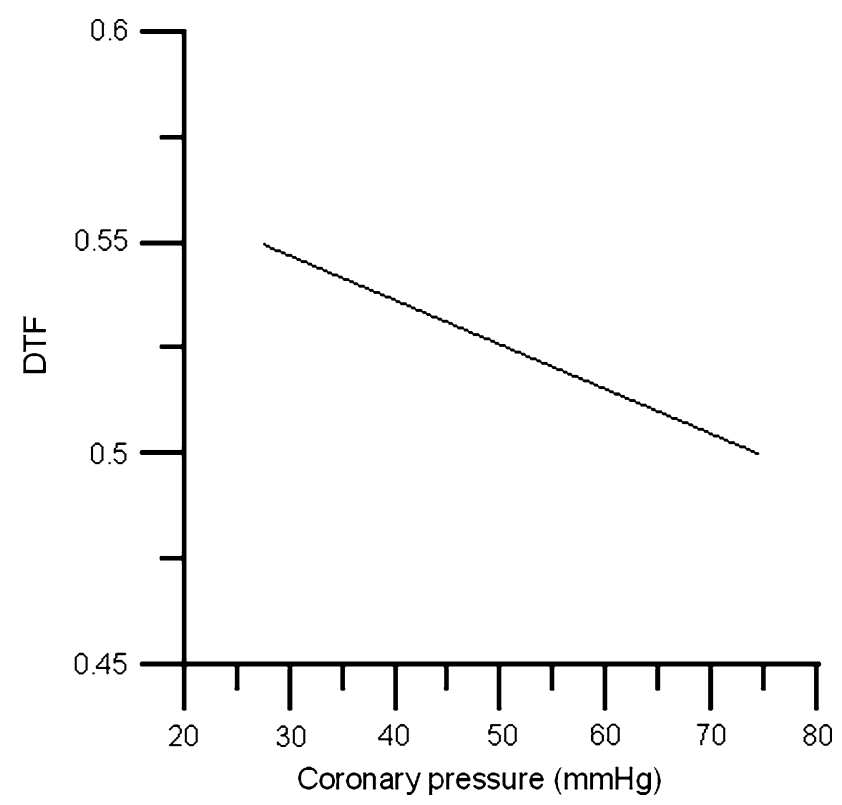

Fig. $6 \mathrm{DTF}_{1}$ as a function of coronary pressure. Please note $\mathrm{DTF}_{1}$ is not defined for the entire range of pressures 

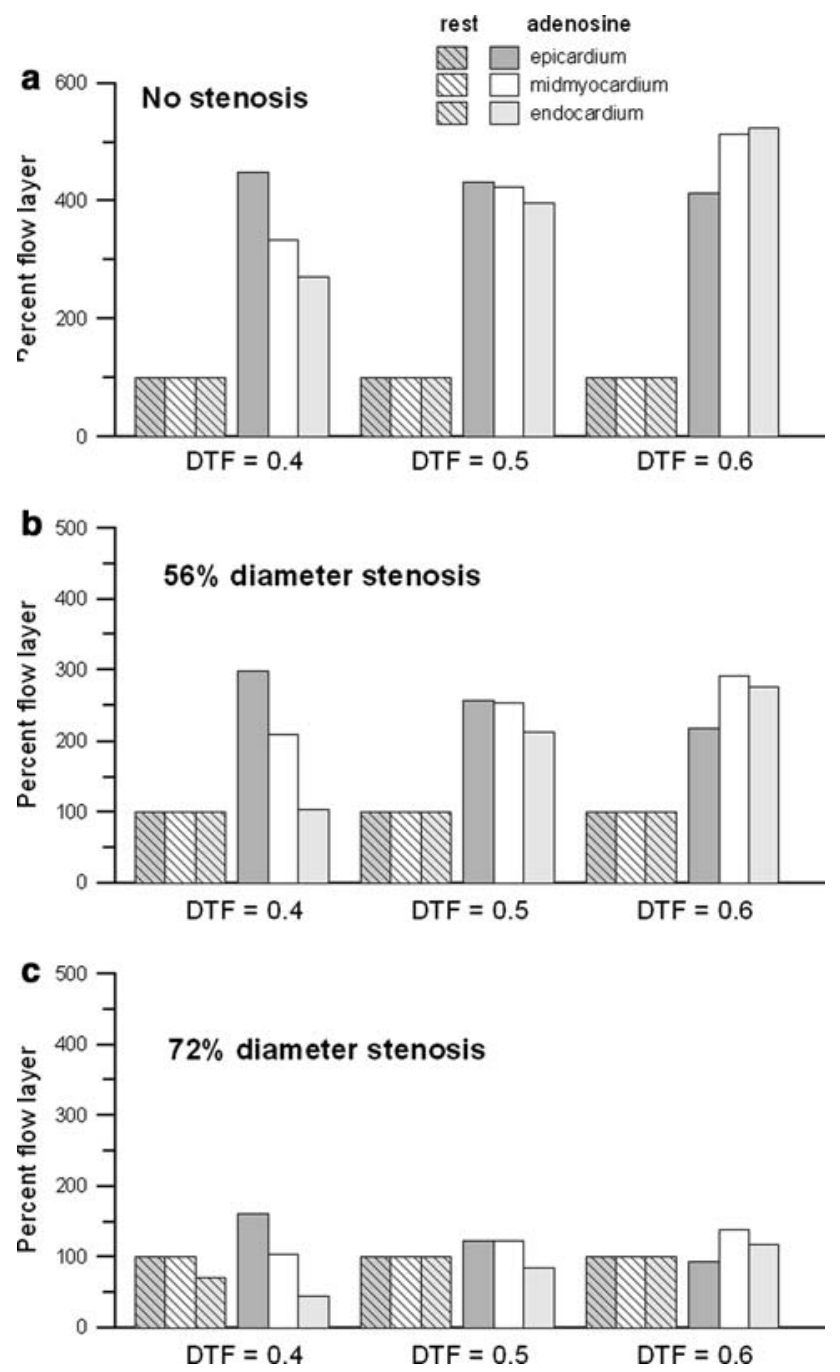

Fig. 7 This figure illustrates that for a mild stenosis, (56\% occlusion), changes in DTF do not result in endocardial underperfusion. However, with a more severe stenosis present ( $72 \%$ occlusion) in absence of normal autoregulation following the administration of adenosine, the flow to the entire myocard may be enhanced at the expense of reduced endocardial flow

whereas the hyperemic flow values depend on DTF and layer type. For the reference value of DTF $=0.5$ hyperemic flow is rather constant for both stenosis conditions. For the $72 \%$ stenosis, hyperemic flow in the subendocardial layer is lower than in the subepicardium. Note that adenosine increases subepicardial flow but reduces subendocardial flow and induces subendocardial ischemia. This difference in layer response is even larger for lower DTF values where subendocardial flow was already below flow demand in the presence of autoregulation.

The drop in flow in one layer related to vasodilation in all layers is referred to as coronary steal. It is the result of increased pressure drop over the stenosis following vasodilatation in the layers with some reserve, i.e., autoregulatory resistance, left. Note that in Fig. 7c for
$\mathrm{DTF}=0.6$ the steal works in the opposite direction and subendocardial flow increases upon adenosine administration at the expense of a reduced subepicardial flow.

\subsection{Increase of flow demand by exercise}

During exercise regional vasodilation is related to regional increased oxygen demand and may become exhausted at either endocardial or epicardial layer, first depending on DTF. This is illustrated by Fig. 8, where the ratio between endocardial and epicardial flow is plotted as a function of normalized flow demand (NFD), with the value 1 corresponding to oxygen demand at rest. Hence, the normalized flow demand is proportional to the change in fractional oxygen consumption change.

For the reference case (no stenosis, PAorta $=90 \mathrm{~mm}$ $\mathrm{Hg}$, DTF $=0.5$ ) represented by a solid line, the relationship is horizontal and ends at the designed maximal flow where all autoregulatory resistances have become 0 corresponding to a normalized flow of about four for each layer. Subepicardial flow in this case is slightly increased as compared to subendocardial flow. In the absence of a stenosis and DTF $=0.4$ the subendocardial autoregulation is exhausted first and endocardial/epicardial flow ratio will start to fall at a normalized flow demand of 2.7. For $\mathrm{DTF}=0.6$ it is the subepicardium where autoregulation is exhausted first but at a higher normalized flow demand.

In the presence of a stenosis this model behavior remains but now the endocardial/epicardial flow ratio cannot be maintained at 1.15 for the entire range but will start to drop at lower normalized flow demand values: at

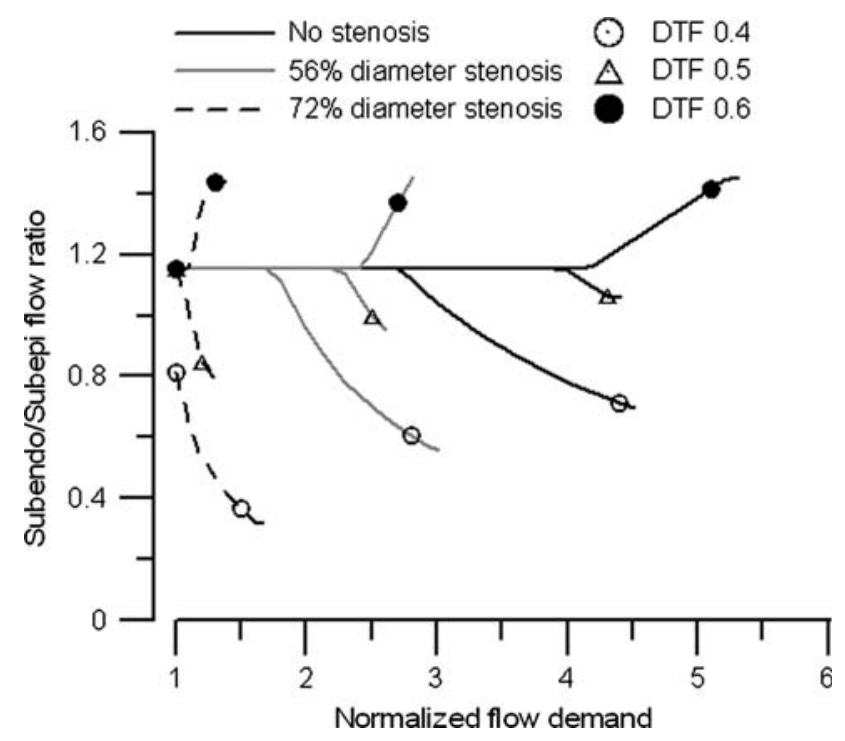

Fig. 8 Ratio of subendo and subepicardial flow as a function of normalized flow demand for different values of DTF 
the reference DTF for $56 \%$ diameter stenosis at NFD $=2.3$ and for $72 \%$ diameter stenosis at NFD $=1$. However, for $\mathrm{DTF}=0.6$ it is the subepicardium that is underperfused first. For DTF $=0.4$ the subendocardium is already severely underperfused for $\mathrm{NFD}=1$, which is only worsened with increasing flow demand.

\subsection{Modulation of stenosis effect on myocardial perfusion by DTF}

Figure 9 provides a graphical analysis of the interaction between hyperemic myocardial perfusion and stenosis hemodynamics related to two often-used clinical indices, FFR and CFVR. The top panel relates to the whole heart and the bottom panel to the subendocardium. Myocardial flow is expressed per gram tissue. The curves related to tissue perfusion are similar to those depicted in Fig. 3. The curves depicting the stenosis hemodynamics are similar to those of Fig. 6, but now the axes are switched and the pressure drop is plotted with respect to aortic pressure. The intersection of a perfusion and stenosis relationship forms defines the operating point at full vasodilation.

It is clear that for a given stenosis a change in DTF shifts the tissue perfusion curve and thereby the intersection with the stenosis relationship. This reflects the reduction in pressure drop over the stenosis when flow is reduced by increasing microvascular resistance. The change in DTF induces changes also in FFR and CVFR for the same stenosis degree. When decreasing DTF from 0.5 to 0.4 FFR increases from 0.75 to 0.79 , which usually is seen as indicative of reduction in stenosis significance while practically the range for subendocardial flow reserve is reduced to the bare minimum. Obviously these responses are even more serious for the higher stenosis degree.

\section{Discussion}

In this model study, we demonstrate the dependence of subendocardial layer perfusion on DTF. Since perfusion of the myocardial layers depends on DTF, clinical measurements that are performed by artificial hyperemia by infusing adenosine may overestimate subendocardial layer perfusion at exercise when DTF is reduced as compared during clinical measurement.

This model study demonstrates the importance of standardization of conditions of cardiac function in testing the physiological state of the coronary circulation. More specifically, cardiac function assessment by using a hyperemia stress test is dependent on myocardial testing conditions. This is especially important for these factors that dominate subendocardial perfusion: DTF and perfusion pressure. The
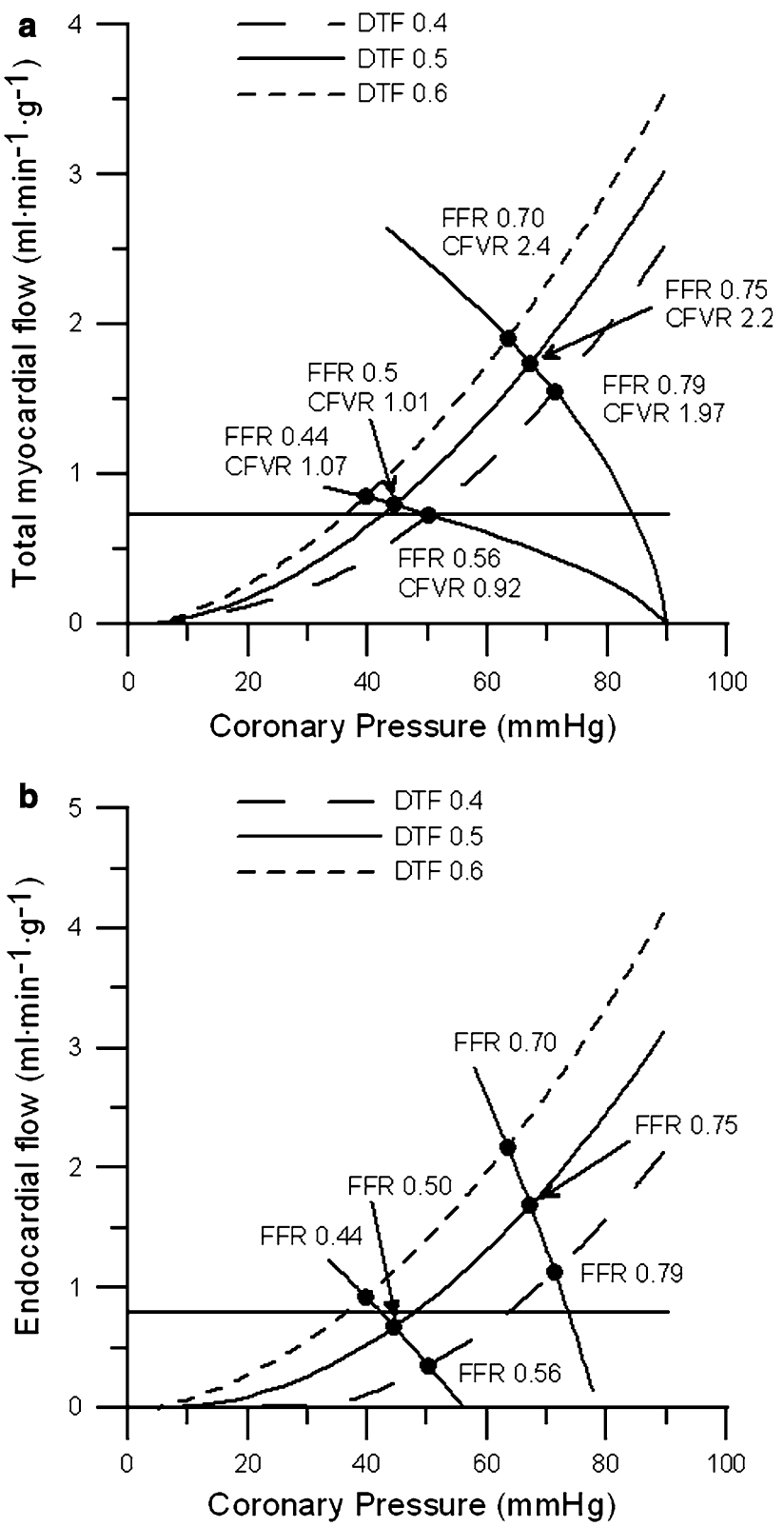

Fig. 9 a Top. Model results for flow pressure curves for different values of DTF for the myocardium as a whole and $\mathbf{b}$ Bottom for the subendocardium alone. Flow is expressed per gram tissue. Note that FFR and CFVR are not constant for a stenosis degree but vary by DTF

absolute values of the perfusion predictions depend on parameterization of the model for which animal data were used. Therefore, extrapolation of distribution of perfusion in the human has to be performed with care. However, the model behavior is rather general and can be interpreted in the following way. There is a reference condition, $\mathrm{DTF}_{1}$, defined by coronary perfusion pressure and DTF for which perfusion of the myocardial layers is evenly distributed. Increasing DTF from this reference value will increase hyperemic subendocardial perfusion while decreasing DTF 
will do the opposite. It further may be concluded that a clinical test applying, e.g., adenosine to obtain maximal vasodilatation may deviate from perfusion distribution during real exercise induced hyperemia.

\subsection{Microsphere data}

The literature contains numerous studies in which microspheres have been applied to study blood flow distribution over the myocardium in hyperemic conditions. The general conclusion is that the subendocardium is the location where blood flow is most sensitive to contraction-related flow-impeding mechanisms [22]. In contrast, perfusion of the subepicardium is hardly or oppositely affected by cardiac contraction [8]. Therefore, the Endo/ Epi flow ratio has been used as an index to define the perfusion state of the subendocardium with the subepicardium as reference.

In experiments where coronary perfusion pressure is maintained, but the heart arrested the endo/epi ratio is about 1.5 [6], which indicates that intrinsic subendocardial vascular resistance (no effect of contraction) is lower than that in the epicardium [23]. This agrees with the observation that luminal volume percentage of resistance arteries in the subendocardium is higher than that at the subepicardium [23]. At very high HR, around 180 beats/min, the endo/epi ratio is about 0.5 . Hence, with rather constant epicardial resistance the contraction of the heart may vary the subendocardial resistance by a factor of three.

For model purposes however, a data set is needed under normal controlled conditions and with sufficient variation in the parameter values for parameter estimations. Few studies do deliver this information. The classical dataset from Bache [1] provides the effect of DTF on hyperemic flow distribution but only at $\mathrm{Pc}=100 \mathrm{~mm} \mathrm{Hg}$. However, it also provides information on flow distribution at rest and hence allows for the estimation of $R_{\text {hyp }}$ and $R_{\text {auto }}$ at this value of Pc. The more recent dataset of Fokkema et al. [8] provides the information on how the relation between $R_{\text {hyp }}$ and DTF is modulated by Pc, essential for prediction of the effects of a stenosis.

\subsection{Sensitivity analysis}

In order to validate the dependency of the model outcomes on the starting parameters a sensitivity analysis was performed. Setting the normal flows for the subendocardium and the subepicardium to the flow level of the midmyocardium did not alter the model outcomes significantly. Setting subepicardial conductance independent of Pc [8], results in the percent flow of the epicardial layer decreasing linear with decreasing Pc. Subepicardial steal in case of a severe coronary stenosis however remained.

Changes in the myocardial layer conductances affect the model outcomes to a larger extent. Increasing the influence of DTF on myocardial layer conductance of midmyocardial and subendocardial layers by $25 \%$, produces comparable outcomes albeit with increased effect of subepicardial steal when DTF is low. Instead, reducing the influence of DTF by $25 \%$ decreases subepicardial steal for low DTF; however, this mechanism remains present. Increasing the influence of myocardial layer perfusion pressure on layer conductance by $25 \%$ leads to lower perfusion when coronary pressure is reduced. In contrast, decreasing the influence of layer perfusion pressure leads to slightly lower pressures when subendocardial perfusion is decreased.

Removal of the Bernoulli component of the SR, in Eq. 1, leads to a decreased luminal diameter, i.e., 67 and $77 \%$ diameter occlusion, necessary for an FFR of 0.75 and 0.5, respectively. Although in this case, the nonlinear pressure loss for severe stenosis was included in the model, it is not essential for interpretation of the model outcomes. Here, different values of DTF, lead to different myocardial layer conductances, so that myocardial flow is altered and as a consequence pressure loss across the stenosis is affected. Finally, the normal flow and conductance of the midmyocardial and subendocardial layers was set equal, and the influence of DTF on layer conductance was reduced. In spite of these changes in model parameters, the model outcomes essentially remain unchanged; i.e., subepicardial hyperemic steal develops for low DTF with adenosine administration and FFR is dependent on DTF.

\subsection{Limitations}

The model we used is empirical and the dependence of the $R_{\text {hyp }}$ on DTF is not based on physical principles of compressed intramural vessels. Several of these models relate the dynamic changes of diameter of intramural vessels to changes in their resistances and predict in that way the effect of DTF and Pc [17]. This latter approach is certainly useful in order to arrive at a more detailed understanding of how contracting myocardium affects its perfusion [22]. However, the present model allows extrapolation from relationships obtained in animal studies to the clinical situation without assumptions related to the actual perfusion contraction interaction.

The experimental data used to parameterize the model were obtained in controlled conditions applying anesthesia [1] and an extra corporeal perfusion system [8]. Most likely the parameters of $R_{\text {hyp }}(\mathrm{DTF}, \mathrm{Pc})$ may differ between man and animals. Therefore, human studies are needed to arrive at reliable parameters. However, it is unlikely that the 
general trends of the model behavior will drastically change and likely that a reference condition under which flow distribution over endocardial and epicardial layers is even will hold. For DTF values lower than the DTF value at this reference condition the subendocardium is at risk but it is relatively safe for higher DTF values.

In this study we kept aortic pressure constant and at $90 \mathrm{~mm} \mathrm{Hg}$ as to stay close to the range of experimental data on flow distribution. It will be especially interesting to see what happens during more pathophysiological conditions as hypertension and hyperthrophy.

The distinction between hyperemic and autoregulatory resistance is very practical for the model design but rather artificial. Autoregulation is caused by changing the smooth muscle tone in small arteries with diameters varying between 10 and $400 \mu \mathrm{m}$. Hyperemic conditions occur when this tone is reduced to 0 and the vessels obtain their maximal diameter determined by the connective tissue in the wall. Hence, in this condition where $R_{\text {auto }}=0$ these vessels still have resistance which contributes to our hyperemic resistance parameter $R_{\text {hyp }}$. Hence, the distinction between $R_{\text {auto }}$ and $R_{\text {hyp }}$ does not relate to anatomical location, but is to be considered as a functional model characteristic that describes the experimental data.

\subsection{Clinical implications}

Currently, MRI-based methods for routine measurements of myocardial perfusion distribution are being developed. It is therefore of paramount importance that the clinician is able to interpret the data resulting from such a new functional imaging techniques. An important benefit is that the spatial resolution of these novel image-based flow distribution modalities, approach that of the earlier microsphere studies in animals. Therefore, a model able to describe quantitatively the earlier findings on perfusion distribution obtained by that technique could be of great help. The present model demonstrates how important it is to standardize the conditions of measurement with respect to DTF and coronary perfusion pressure.

In general it is recognized that the subendocardium is most vulnerable to ischemia and this has been related to the increased subendocardial resistance as a result of heart contraction. This model study demonstrates that this paradigm needs modification since the vulnerability, expressed as reduced hyperemic subendocardial flow depends on DTF and perfusion pressure.

Patients with signs of cardiac ischemia are in general medicated with Beta-blockers in order to reduce their heart rate and systemic blood pressure. Two important beneficial effects then result; myocardial oxygen demand is reduced, and the DTF-related hyperemic flow potential at the subendocardium is increased. The positive effect of reduction in oxygen demand is better recognized than the effect of increased hyperemic flow potential. However, it can be shown that the sensitivity of hyperemic flow potential to a change in HR is larger than the sensitivity to a change in oxygen demand [17].

Exercise-induced hyperemia is difficult to realize during catheterization or MRI measurement. Therefore, adenosine is administered to induce maximal vasodilation and thereby hyperemic conditions. However, the present model clearly demonstrates that in the presence of a stenosis pharmacological vasodilation has different effects on the heart than exercise-induced vasodilation. Pharmacological vasodilation augments 'steal' of perfusion from one layer to another and depending on DTF can favor perfusion of the subendoardium or subepicardium.

During catheterization the physiological severity of a stenosis is often indicated by the FFR. Assuming that the venous pressure is 0 , FFR simply is the ratio between pressure distal of the stenosis and aortic pressure but measured at hyperemic conditions where all autoregulatory resistances are 0 . In terms of resistance circuit analysis such as depicted in Fig. 1b, this ratio equals $R_{\mathrm{m}} /$ $\left(R_{\mathrm{s}}+R_{\mathrm{m}}\right)$ where $R_{\mathrm{m}}$ is the replacement of all distal resistances in parallel and $R_{\mathrm{S}}$ is the SR. Consequently, FFR only reflects $R_{\mathrm{s}}$ when $R_{\mathrm{m}}$ is well-defined. In the clinical literature it is often assumed that $R_{\mathrm{m}}$ is constant but this model study indicates clearly that $R_{\mathrm{m}}$, and therefore FFR, depends on DTF and not uniquely reflects the SR. This dependency is not unimportant since in clinical practice a threshold of FFR $=0.75$ is applied below which a stent is placed. Hence, a change in HR during the measurement of FFR may result in a change of treatment decision [16].

Although FFR and CFVR depend on DTF, the changes in these epicardial-determined indices reflect only the changes in subendocardial perfusion in a rather attenuated manner as indicated by Figs. 9a and b. Obviously, this is the result of the subepicardial layer which resistance is rather independent of DTF and blunts the response of the subendocardium on epicardial measurements. Using a SPECT stress test, threshold values for FFR $=0.75$ and $\mathrm{CFVR}=2$ have been reported in the literature $[9,11,13]$ below which ischemia is induced. Figure $9 \mathrm{~b}$ demonstrates that these values correspond to the reference value chosen for this study where the endocardial epicardial flow ratio is 1 at a DTF $=0.5$. Based on this figure one might conclude that at FFR $=0.75$ a flow reserve is left of about 2 . However, this flow reserve will be lost with an increase in oxygen consumption that most likely is the result of an increased HR inducing a downward shift in the subendocardial hyperemic flow pressure relationship. As a consequence, pharmacologically determined flow reserve, 
without simultaneously increased oxygen demand, may prove an overestimation of the actual increase in oxygen demand that the coronary circulation may facilitate.

An important clinical implication of this study is the finding of epicardial hyperemic steal [2, 4, 7], where flow is increased in the epicardium at the expense of a decrease in the subendocardium. Figure 7 illustrates that upon administration of adenosine the autoregulatory resistances are set to 0 , after which flow distribution to the myocardium may change significantly. These changes in flow distribution may especially be significant when due to a coronary stenosis the endocardial autoregulation resistance prior to adenosine administration was absent or reduced [10]. In that case endocardial flow may significantly decrease due to decreased coronary pressure, which is reduced as a consequence of increased flow to the entire myocardium through the stenosis after adenosine administration. This effect is illustrated in Fig. 7c.

In the case that microvascular dysfunction might have significantly increased the hyperemic resistance [5], an increased myocardial flow by lowering of autoregulatory resistances, may cause abnormal distribution of flow and result in endocardial ischemia [12]. This type of flow redistribution in hyperemia, has been suggested with the use of MRI [12, 19]. However, the reduction in endocardial perfusion was not confirmed in a recent study [19], but instead the ratio of endocardial and epicardial flow was reduced. The second effect here, precluding an accurate assessment of endocardial flow is the influence of DTF. As established previously, changes in DTF may change hyperemic resistance significantly; causing either adequate endocardial perfusion or significant underperfusion as is illustrated by Figs. 9a and $\mathrm{b}$.

This study also confirms the beneficial action of drugs that prolong DTF, e.g., dobutamine [3, 8] without affecting HR, or propranolol, a beta-blocker which increases DTF and reduces HR [3], or isoproterenol which is a beta adrenergic agonist similar to dobutamine and increases DTF by shortening systole while increasing HR [3].

\section{Conclusions}

In conclusion, using a simple model of the coronary circulation with hyperemic resistances dependent on DTF and coronary pressure, the concept of epicardial hyperemic steal of flow at the expense of endocardial flow is illustrated. This hyperemic steal is especially influential when endocardial autoregulation is low as a consequence of reduced coronary pressure, and may preclude an accurate clinical assessment of endocardial perfusion. In addition, for identical stenosis severity, DTF influences the coronary pressure, implying in our model that DTF alters the measured FFR ratio for identical stenosis and aortic pressure. These results may bear highly significant importance for the adenosine-assisted evaluation of subendocardial underperfusion involved in the treatment of coronary stenosis by interventional cardiology stent placement.

Acknowledgments JPHM van den Wijngaard is supported by the Netherlands Heart Foundation (NHS 2006B226) and in part by a grant from the Niels Stensen Foundation, Amsterdam, The Netherlands.

\section{References}

1. Bache RJ, Cobb FR (1977) Effect of maximal coronary vasodilation on transmural myocardial perfusion during tachycardia in the awake dog. Circ Res 41:648-653

2. Bache RJ, Cobb FR, Greenfield JC Jr (1974) Myocardial blood flow distribution during ischemia-induced coronary vasodilation in the unanesthetized dog. J Clin Invest 54:1462-1472

3. Boudoulas H, Rittgers SE, Lewis RP, Leier CV, Weissler AM (1979) Changes in diastolic time with various pharmacologic agents: implication for myocardial perfusion. Circulation 60:164-169

4. Buckberg GD, Fixler DE, Archie JP, Hoffman JI (1972) Experimental subendocardial ischemia in dogs with normal coronary arteries. Circ Res 30:67-81

5. Camici PG, Crea F (2007) Coronary microvascular dysfunction. N Engl J Med 356:830-840

6. Domenech RJ (1978) Regional diastolic coronary blood flow during diastolic ventricular hypertension. Cardiovasc Res 12:639-645

7. Domenech RJ, Goich J (1976) Effect of heart rate on regional coronary blood flow. Cardiovasc Res 10:224-231

8. Fokkema DS, VanTeeffelen JW, Dekker S, Vergroesen I, Reitsma JB, Spaan JA (2005) Diastolic time fraction as a determinant of subendocardial perfusion. Am J Physiol Heart Circ Physiol 288:H2450-H2456

9. Kern MJ, Lerman A, Bech JW, et al. (2006) Physiological assessment of coronary artery disease in the cardiac catheterization laboratory: a scientific statement from the American Heart Association Committee on Diagnostic and Interventional Cardiac Catheterization, Council on Clinical Cardiology. Circulation 114:1321-1341

10. Merkus D, Vergroesen I, Hiramatsu O, et al (2001) Stenosis differentially affects subendocardial and subepicardial arterioles in vivo. Am J Physiol Heart Circ Physiol 280:H1674-H1682

11. Meuwissen M, Siebes M, Chamuleau SA, et al (2002) Hyperemic stenosis resistance index for evaluation of functional coronary lesion severity. Circulation 106:441-446

12. Panting JR, Gatehouse PD, Yang GZ, et al (2002) Abnormal subendocardial perfusion in cardiac syndrome $\mathrm{X}$ detected by cardiovascular magnetic resonance imaging. $N$ Engl J Med 346:1948-1953

13. Pijls NH, Van Gelder B, Van d V et al (1995) Fractional flow reserve. A useful index to evaluate the influence of an epicardial coronary stenosis on myocardial blood flow. Circulation 92:3183-193

14. Siebes M, D’Argenio DZ, Selzer RH (1985) Computer assessment of hemodynamic severity of coronary artery stenosis from angiograms. Comput Methods Programs Biomed 21:143-152

15. Siebes M, Chamuleau SA, Meuwissen M, Piek JJ, Spaan JA (2002) Influence of hemodynamic conditions on fractional flow 
reserve: parametric analysis of underlying model. Am J Physiol Heart Circ Physiol 283:H1462-H1470

16. Siebes M, Verhoeff BJ, Meuwissen M, de Winter RJ, Spaan JA, Piek JJ (2004) Single-wire pressure and flow velocity measurement to quantify coronary stenosis hemodynamics and effects of percutaneous interventions. Circulation 109:756-762

17. Spaan JA (1991) In: Spaan JA (ed) Coronary blood flow, 1st edn. Kluwer, Dordrecht

18. Spaan JA, Piek JJ, Hoffman JI, Siebes M (2006) Physiological basis of clinically used coronary hemodynamic indices. Circulation 113:446-455

19. Vermeltfoort IA, Bondarenko O, Raijmakers PG, et al (2007) Is subendocardial ischaemia present in patients with chest pain and normal coronary angiograms? A cardiovascular MR study. Eur Heart J 28:1554-1558
20. Vogel R, Indermuhle A, Reinhardt J, et al (2005) The quantification of absolute myocardial perfusion in humans by contrast echocardiography: algorithm and validation. J Am Coll Cardiol 45:754-762

21. Westerhof N, Stergiopulos N, Noble MIM (2005) In: Nico Westerhof, Nikos Stergiopulos, Mark IM Noble (eds) Snapshots of hemodynamics, 1st edn. Springer, New York

22. Westerhof N, Boer C, Lamberts RR, Sipkema P (2006) Cross-talk between cardiac muscle and coronary vasculature. Physiol Rev $86: 1263-1308$

23. Wusten B, Buss DD, Deist H, Schaper W (1977) Dilatory capacity of the coronary circulation and its correlation to the arterial vasculature in the canine left ventricle. Basic Res Cardiol 72:636-650 